\title{
Not All STEMI Patients Receive Timely Reperfusion: Considerations for Rural Emergency Departments
}

\author{
Helen Orvad (D) \\ Lindsay Savage ${ }^{2}$ \\ Tony Smith (iD ${ }^{3}$ \\ Mohammad Hamiduzzaman (iD ${ }^{3}$ \\ David Schmidt (D) ${ }^{4}$ \\ 'Hunter New England Local Health \\ District, Tamworth, NSW, Australia; \\ ${ }^{2}$ Hunter New England Local Health \\ District, Newcastle, NSW, Australia; \\ ${ }^{3}$ University of Newcastle Department of \\ Rural Health, Taree, NSW, Australia; \\ ${ }^{4}$ Health Education and Training Institute, \\ Australia Health Education and Training \\ Institute, Sydney, NSW, Australia
}

Correspondence: Tony Smith University of Newcastle Department of Rural Health, Taree, NSW, 2430, Australia Tel +6I+2+4055 I912

Email tony.smith@newcastle.edu.au

\begin{abstract}
Early reperfusion for ST-elevation myocardial infarction (STEMI) is well known to improve patient outcomes. A review of patient records in one rural health service in New South Wales, Australia, suggested that not all STEMI patients were receiving timely reperfusion. Consequently, the aim of this study was to further investigate factors influencing clinical decision making by primary care providers in relation to rural STEMI patients. This cross-sectional observational study was in two phases, a retrospective audit of patient records and a survey of rural general practitioners (GPs). In the first phase, patients with STEMI who were referred from small rural hospitals to a regional hospital emergency department (ED) were identified through the local health district database. In phase two, information from the database informed questions for a survey distributed to the GP visiting medical officers (VMOs) at small rural hospitals in the region. The survey was designed to ascertain factors that may contribute to delays in the care of STEMI patients. Of the STEMI patients identified $(\mathrm{n}=139), 15 \%$ (21) who were eligible for medical reperfusion were not administered thrombolysis within 4 hours of triage. Auditing of this group's records found that ECGs were inaccurately interpreted for $76 \%$ of the missed STEMI patients. In the survey, about $55 \%$ of the GP respondents said they "very much agree" with the statement that they felt competent in STEMI management. Only 64\% of the GP VMOs agreed they felt competent in diagnosis and management of a failed thrombolysis and not all respondents were aware of the relevant clinical guideline. Patients with missed STEMI are at higher risk of morbidity and mortality and increased length of stay, adding burden to the patient, carer and health service. Without addressing gaps in service provision and better adherence to clinical guidelines, unacceptable delays in STEMI management in rural health services are likely to continue.
\end{abstract}

Keywords: coronary care, rural, emergency, thrombolysis

\section{Introduction}

The disparity between ST-Elevation Myocardial Infarction (STEMI) patients' management guidelines and actual clinical practice has been highlighted previously, ${ }^{1}$ but it remains unclear how clinical practice gaps manifest in relatively small rural emergency departments (EDs). The delayed management of STEMI is associated with increased morbidity and mortality, ${ }^{2}$ resulting from occluded arteries not being reopened (reperfusion) either by percutaneous coronary intervention (PCI) or intravenous fibrinolytic therapy (thrombolysis). ${ }^{2}$ While PCI is associated with lower mortality, non-fatal reinfarction and stroke, ${ }^{3}$ in rural EDs, remote from large 
regional hospitals, reperfusion is by thrombolysis, as access to an angiographic facility is not immediate, if possible at all. Patients with missed STEMI or those not managed according to recommended guidelines are at increased risk of lengthy hospital stay, which in turn adds burden to the patients, carers and health services.

\section{STEMI Management Guidelines}

In Australia, the National Heart Foundation of Australia and Cardiac Society of Australia and New Zealand's Clinical Guidelines for Management of Acute Coronary Syndrome $(\mathrm{ACS})^{4}$ and the Australian Commission on Safety and Quality in Health Care's ACS Clinical Care Standard ${ }^{5}$ define minimum care requirements for STEMI patients. Other countries have similar guidelines. ${ }^{6}$ To commence timely STEMI management, patients must be triaged as a minimum category 2 and should have an electrocardiogram (ECG) performed and reviewed by a competent physician within 10 minutes of arrival in ED. The Minimum Standards for Chest Pain Evaluation of the New South Wales (NSW) Ministry of Health (MoH) $(2011)^{7}$ provide an evidence-based clinical tool that directs the patients' care with ACS in ED, including triage, risk stratification, diagnostic test recommendations, timelines, and medication dosages. The more recent NSW $\mathrm{MoH}$ Pathway for Acute Coronary Syndrome Assessment $(\mathrm{PACSA})^{8}$ is specifically designed for use by all remote, rural and metropolitan health services and contains relevant checklists and flowcharts.

\section{Issues in Rural STEMI Management}

To illustrate the issues and challenges at small rural hospitals in one region of New South Wales, Australia, this article incorporates the findings of a retrospective clinical audit of STEMI patients transferred to the tertiary referral hospital from smaller outlying hospitals. Also included below are results from a survey of GP Visiting Medical Officers (VMOs) at small rural hospitals about factors that they perceived may delay the management of STEMI patients. Ethics clearance for the combined clinic audit and survey was received from the relevant Hunter New England Human Research Ethics Committee [Reference No: 2018/ETH00643; December 2018]. Data access complied with the National Health and Medical Research Council National Statement on the Conduct of Research on Humans, as updated in 2018.

\section{Clinical Audit}

To better appreciate the clinical management of rural STEMI patients, medical records were audited for 2016 and 2017 of patients who presented to rural EDs at small rural hospitals in the region and were subsequently transferred to the referral hospital. The aim was to identify misdiagnosed STEMI patients and establish common variables, including demographic or locational factors, results of diagnostic tests, duration of hospitalisation or readmission, and apparent delays in treatment. The audit was conducted by a Clinical Nurse Consultant in Cardiology. Complex STEMI ECG diagnoses were blind-reviewed by a Consultant Cardiologist for confirmation that ECG changes were clinically significant relative to treatment options.

There were 139 patients who met the eligibility criteria and who had a STEMI confirmed by ECG, of which $57 \%$ were males and the average age was 69 years. One of these patients identified as being of Aboriginal or Torres Strait Islander origin. The patients were transferred from hospitals in 21 different towns in the region, classified according to the Australian Statistical Geographical Standard Remoteness Area ${ }^{9}$ as RA3 (Outer Regional) $(n=18)$ or RA2 (Inner Regional) ( $n=3)$. The population size of the RA3 towns ranged between about 1150 and 7400, while all the RA2 towns had greater than 25,000 residents.

Twenty-one of the patients (15\%) were considered to have a "missed STEMI", which was defined as not having been diagnosed as having had a STEMI within 4 hours of their initial ED presentation, despite being eligible for thrombolysis. This excluded patients who had earlydocumented absolute or relative contraindications for thrombolysis and were not eligible for active treatment $(\mathrm{n}=6)$, or those who had an explainable delay $(\mathrm{n}=2)$. Patients were also excluded who had a complex STEMI ECG that the Consultant Cardiologist considered beyond the interpretive capabilities of most rural GPs $(n=2)$.

Data showed that 13 of the "missed STEMI" patients (64\%) were not seen in ED by a GP for 15 minutes or more after their initial presentation and for 16 patients (76\%) their initial ECG was misinterpreted. With $18 \%$ of patients in the audit having been triaged as category 3 or 4 , the response time was delayed for those patients. Figure 1A shows clinical outcomes for the 21 patients who were initially misdiagnosed, for whom the mean length of stay in hospital initially was 5.4 days, while 8 $(38 \%)$ were readmitted within 28 days with a cardiac related condition. 


\section{Survey Findings}

The survey was performed to explore the factors influencing clinical decision-making in patients suspected of STEMI. The survey was conducted by the first author in March 2019, with advice and assistance from the coauthors. Of the 66 questionnaires posted to GP VMOs in the region, $22(33 \%)$ were completed and returned. Questions explored the participants' self-perceptions of their competency in managing STEMI patients, as well as their perceptions of support they received. The questionnaire consisted of 20 statements, with responses on a 5-point scale: "Very much agree"; "Somewhat agree"; "Neither agree or disagree"; "Somewhat disagree"; or "Do not agree". In addition, space was provided for an openended comment. The list of questions is included as a Supplementary File.

About $55 \%$ of the GP respondents said they "Very much agree" with the statement that they felt competent in STEMI management and a further $41 \%$ said they "Somewhat agree"; yet, for $76 \%$ of the missed STEMI patients whose records were audited, ECGs were inaccurately interpreted, which potentially delayed their

\section{A Clinical Outcomes of Missed STEMI Patients}

$$
(\mathrm{n}=21)
$$

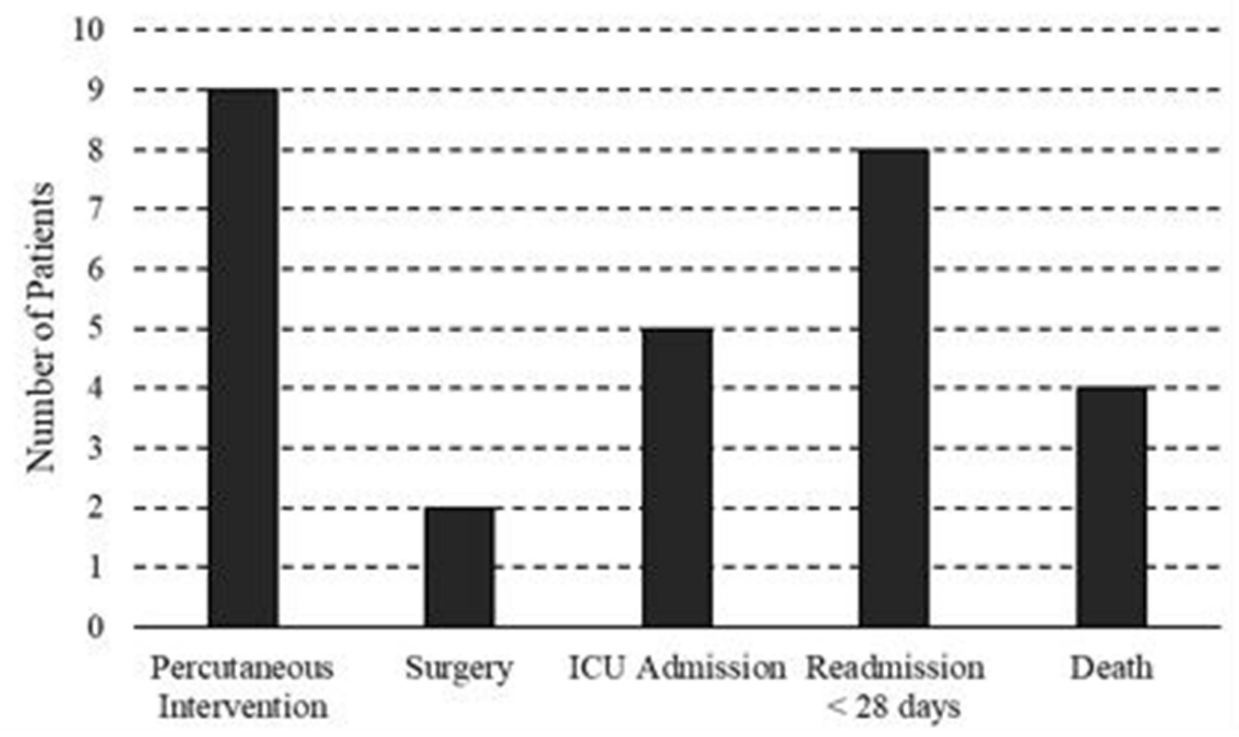

\section{B Rural GP Awareness \& Use of Prescribed Chest Pain Pathway}

$$
(\mathrm{n}=22)
$$

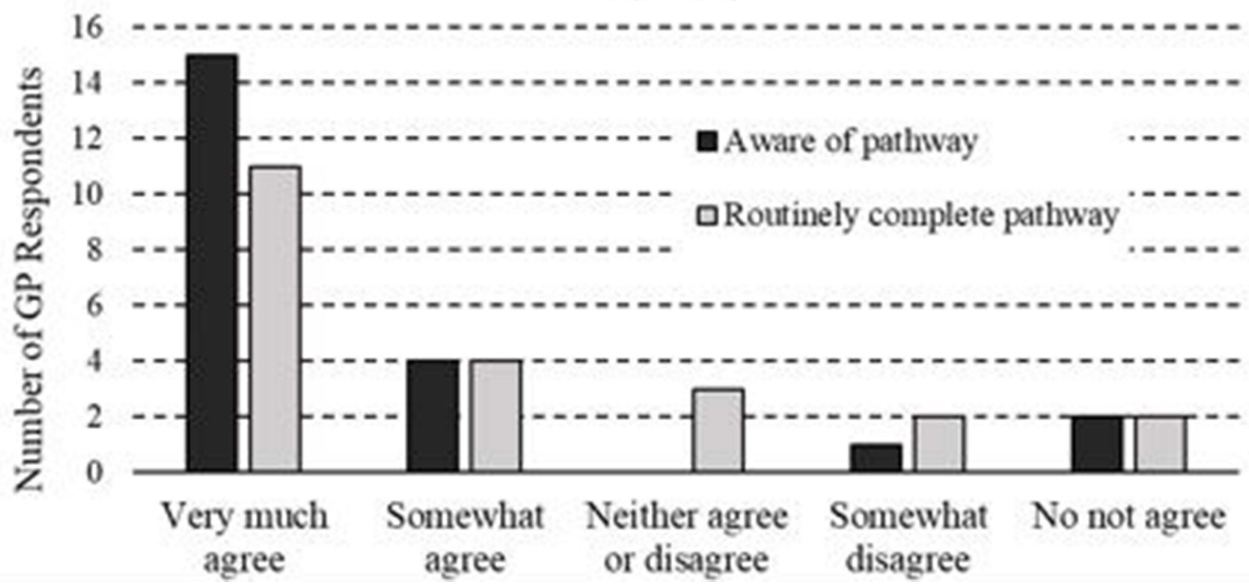

Figure I (A) Outcomes for patients not initially diagnosed with a STEMI at rural hospitals and, (B) responses from GP VMOs about awareness and use of the chest pain clinical pathway. 
progression to definitive care. Only 64\% of the GP VMOs agreed they felt competent in diagnosis and management of a failed thrombolysis. As shown in Figure 1B, 15 respondents (68\%) "Very much agreed" and $4(18 \%)$ "Somewhat agreed" that they were aware of the NSW $\mathrm{MoH}$ chest pain pathway, with the remaining 3 disagreeing with that statement. In response to whether they routinely used that clinical pathway, however, a smaller proportion either "Very much" or "Somewhat agreed", suggesting a significant knowledge and practice gap for some. Only $23 \%$ "Very much agreed" that they felt competent in diagnosing a posterior STEMI. Meanwhile, $86 \%$ of respondents felt equally supported by local nursing staff and the staff at the referral hospitals, but only $77 \%$ agreed they felt supported by medical colleagues at their own hospital.

\section{Challenges for Small Rural Hospitals}

While the sample size is small and confined to one region, the audit and survey raised questions about accurate diagnosis and treatment of patients with potentially lifethreatening cardiac conditions in smaller rural health-care settings. ${ }^{10}$ Treatment is complicated in smaller rural sites that are isolated from PCI capable facilities and other specialised services. Even with the latest diagnostic equipment, communication technologies and pathways whereby specialist consultation is feasible, there are still diagnostic and treatment challenges, ${ }^{11}$ especially for vulnerable older patients. ${ }^{12}$ The results of the survey suggest that attention was needed to behaviours around clinical decision-making processes, prioritisation of care, and implementation of evidence-based guidelines and collaborative practice.

Previous studies highlight some of the challenges of managing STEMI patients in small rural health-care facilities. A qualitative study in the United States (US) demonstrated the importance of quality improvement initiatives in achieving pre-hospital efficiencies, with a dependency on teamwork, technology and training. ${ }^{13}$ While the findings resonate with the local, Australian experience, especially with regard to the need for greater teamwork, the differences between the Australian and US health-care systems render comparisons difficult. An older US study looked at inter-hospital transfer of rural STEMI patients, principally for the purpose of undergoing PCI, ${ }^{14}$ finding that transfer was feasible, although again, the influence of different health-care environments and transport infrastructure is unclear. In Australia, there is agreement in the literature that management of STEMI of patients in rural locations is problematic, ${ }^{15}$ with disparities evident between rural and metropolitan management and outcomes. ${ }^{16,17}$ This study, however, with its focus on missed STEMIs, calls attention to some particular issues, such as the need for better training in triage for ED nurses, and support for rural GPs in ECG interpretation and the recognition of failed thrombolysis, as well as greater awareness of clinical guidelines.

It is difficult to remain competent in a setting with limited exposure and, in the survey, half of the GP VMOs indicated that they manage STEMI patients less than six times per year. Further, in the busy ED setting, adequate knowledge, interdisciplinary care and timely decision making are not always achievable. Care may be also compromised by some members of the multidisciplinary team not appreciating the urgency and need for timely management in order to avoid further complications or mortality. ${ }^{18}$ This was apparent from the audit in the delays identified in time to senior medical officer review and patient transfer. The finding that some survey respondents did not feel well supported by staff at the larger referral hospitals may also contribute to delayed STEMI management. Literature supports the need for collaboration with cardiology and regional hospital EDs for high-risk patients, where person-centred support is crucial for accurate and timely management. ${ }^{10}$ This is well documented in clinical guidelines and policies, being identified as a priority in the NSW MoH Chest Pain Pathway ${ }^{6}$ and in the more recent PACSA guideline. ${ }^{7}$

\section{Lessons Learned and} Recommendations

This small-scale study points to gaps in use of practice guidelines, clinical knowledge, diagnostic tool use and coordination of support mechanisms in managing patients who present to rural EDs with a suspected STEMI. Addressing these issues would have positive implications for practice and potentially improved patient outcomes. Therefore, some practical considerations for rural EDs are as follows:

- Recurrent, structured education and reassessment of nursing triage knowledge and skills should be implemented in conjunction with First Line Emergency Care (FLEC) training from local health service educators. 
- Emergency medical team education should address the needs of rural GP VMOs to strengthen ECG interpretation competency and STEMI diagnosis, with regular collaboration and follow-up.

- A centralised ECG reading service for the support of rural GPs should be established, thus improving diagnostic accuracy and reducing variability in timely interpretation.

- Debriefing among relevant practitioners after transfer of STEMI patients would improve timely feedback and help build knowledge and confidence of GP VMOs at more remote sites.

\section{Conclusion}

Clinical guidelines list the steps required to provide optimum care of STEMI patients; nevertheless, delays in treatment continue to occur, especially in smaller rural emergency departments. It is hoped that this brief article might encourage others to perform similar clinical audits and surveys that could inform the development of future guidelines in this important practice arena and ultimately expedite access to high-quality care and improve patient outcomes.

\section{Disclosure}

Professor Tony Smith reports grants from NSW Ministry of Health, Health Education and Training Institute, during the conduct of the study. The authors report no other conflicts of interest in this work.

\section{References}

1. Fox KA, Goodman SG, Anderson FA Jr, et al.; GRACE Investigators. From guidelines to clinical practice: the impact of hospital and geographical characteristics on temporal trends in the management of acute coronary syndromes. The Global Registry of Acute Coronary Events (GRACE). Eur Heart J. 2003;24(15):1414-1424. PMID: 12909070. doi:10.1016/s0195-668x(03)00315-4

2. Fazel R, Joseph TI, Sankardas MA, et al. Comparison of reperfusion strategies for ST-segment-elevation myocardial infarction: a multivariate network meta-analysis. $J$ Am Heart Assoc. 2020;9(12): e015186. doi:10.1161/JAHA.119.015186

3. Weaver WD, Cerqueira M, Hallstrom AP, et al. Prehospital-initiated vs hospital-initiated thrombolytic therapy. The Myocardial Infarction Triage and Intervention Trial. JAMA. 1993;270(10):1211-1216. [PMID: 8355383]. doi:10.1001/jama.1993.03510100061033

4. National Heart Foundation of Australia and Cardiac Society of Australia and New Zealand. Australian Clinical Guidelines for the Management of Acute Coronary Syndromes 2016. Heart Lung Circ. 2016;25:895-951. doi:10.1016/j.hlc.2016.06.789
5. Australian Commission on Safety and Quality in Health Care. Acute Coronary Syndromes Clinical Care Standard. Sydney: December 2019. Available from: https://www.safetyandquality.gov.au/sites/ default/files/2019-12/acute_coronary_syndromes_ccs_-_december 2019.pdf. (safetyandquality.gov.au). Accessed 17 August 2021.

6. Ibanez B, James S, Agewall S, et al.; ESC Scientific Document Group. 2017 ESC Guidelines for the management of acute myocardial infarction in patients presenting with ST-segment elevation: the Task Force for the management of acute myocardial infarction in patients presenting with ST-segment elevation of the European Society of Cardiology (ESC). Eur Heart J. 2018;39(2):119-177. PMID: 28886621. doi:10.1093/eurheartj/ehx393

7. New South Wales Department of Health. Minimum Standards for Chest Pain Evaluation: implementation Support Guide. NSW Ministry of Health, Sydney: October 2011. Available: https://aci. health.nsw.gov.au/resources/cardiac/model-of-care/cardiacc-mocs/ min-standards-chest-pain.pdf. (nsw.gov.au). Accessed 17 August 2021.

8. New South Wales Ministry of Health. Pathway for Acute Coronary Syndrome Assessment (PACSA). Sydney: October 2019. Available from: https://www1.health.nsw.gov.au/pds/ActivePDSDocuments/ GL2019_014.pdf. (nsw.gov.au). Accessed 17 August 2021.

9. Australian Government Department of Health. Australian Statistical Geographical Classification-Remoteness Area. Available from: https://www.health.gov.au/health-topics/health-workforce/healthworkforce-classifications/australian-statistical-geographical-classifica tion-remoteness-area. Accessed 11 May 2021.

10. Williams T, Savage L, Whitehead N, Orvad H, Cummins C, Faddy S. Missed Acute Myocardial (MAMI) in a rural and regional setting. Int J Cardiol Heart Vasc. 2019;22:177-180.

11. Koutsoukis A, KanaKakis I. Challenges and answered questions in STEMI management. Hellenic J Cardiol. 2019;60(4):211-215. doi:10.1016/j.hjc.2019.01.001

12. Kayani WT, Khan MR, Deshotels MR, Jneid H. Challenges and controversies in management of ACS in elderly patients. Curr Cardiol Rep. 2020;22(51):254. doi:10.1007/s11886-020-01298-x

13. Carpenter R, McWhorter R, Donaldson S, Silberman D, Maffei S. Working against the clock: a model for rural STEMI triage. Health Services Insights. 2021;14:117863292110375. doi:10.1177/ 11786329211037521

14. Aguirre FV, Varghese JJ, Kelley MP, et al.; Stat Heart Investigators. Rural interhospital transfer of ST-elevation myocardial infarction patients for percutaneous coronary revascularization: the Stat Heart Program. Circulation. 2008;117(9):1145-1152. Epub 2008 Feb 11. PMID: 18268151. doi:10.1161/CIRCULATIONAHA.107.728519

15. Thompson SC, Nedkoff L, Katzenellenbogen J, Hussain MA, Sanfilippo F. Challenges in managing acute cardiovascular diseases and follow up care in rural areas: a narrative review. Int $J$ Environ Res Public Health. 2019;16(24):5126. PMID: 31847490; PMCID: PMC6950682. doi:10.3390/ijerph16245126

16. Nadel J, Hewitt T, Horton D. Acute coronary syndrome in Australia: where are we now and where are we going? AMJ. 2014;7 (3):149-156. doi:10.4066/AMJ.2014.1921

17. Chew DP, French J, Briffa TG, et al. Acute coronary syndrome care across Australia and New Zealand: the SNAPSHOT ACS study. Med J Aust. 2013;199(3):185-191. doi:10.5694/mja12.11854

18. Jarvis S, Saman S. Diagnosis, management and nursing care in acute coronary syndrome. Nurs Times. 2017;113(3):31-35. 


\section{Publish your work in this journal}

The Journal of Multidisciplinary Healthcare is an international, peerreviewed open-access journal that aims to represent and publish research in healthcare areas delivered by practitioners of different disciplines. This includes studies and reviews conducted by multidisciplinary teams as well as research which evaluates the results or conduct of such teams or healthcare processes in general. The journal covers a very wide range of areas and welcomes submissions from practitioners at all levels, from all over the world. The manuscript management system is completely online and includes a very quick and fair peer-review system. Visit http://www.dovepress.com/testimonials. php to read real quotes from published authors. 